Please do not remove this page

RMIT

UNIVERSITY

\title{
Velocity autocorrelation functions of hard-sphere fluids: Long-time tails upon undercooling
}

Williams, Stephen; Bryant, Gary; Snook, Ian; Van Megen, William

https://researchrepository.rmit.edu.au/esploro/outputs/9921862056001341/filesAndLinks?institution=61RMIT_INST\&index=null

Williams, S., Bryant, G., Snook, I., \& Van Megen, W. (2006). Velocity autocorrelation functions of hard-sphere fluids: Long-time tails upon undercooling. Physical Review Letters, 96(8),

087801-1-087801-087804. https://doi.org/10.1103/PhysRevLett.96.087801

Published Version: https://doi.org/10.1103/PhysRevLett.96.087801

Repository homepage: https://researchrepository.rmit.edu.au

(c) 2006 The American Physical Society

Downloaded On 2023/04/26 14:19:46 +1000

Please do not remove this page 


\title{
Velocity Autocorrelation Functions of Hard-Sphere Fluids: Long-Time Tails upon Undercooling
}

\author{
Stephen R. Williams, ${ }^{1}$ G. Bryant, ${ }^{2}$ I. K. Snook, ${ }^{2}$ and W. van Megen ${ }^{2}$ \\ ${ }^{1}$ Research School of Chemistry, Australian National University, Canberra, ACT 0200, Australia \\ ${ }^{2}$ Department of Applied Physics, Royal Melbourne Institute of Technology, Melbourne, Victoria 3000, Australia
}

(Received 16 December 2005; published 28 February 2006)

\begin{abstract}
Molecular dynamics simulations are employed to obtain the velocity autocorrelation function (VAF) for hard spheres, spanning a wide range of volume fractions from dilute to high-density metastable fluids. For all volume fractions below freezing, Alder's classical positive 3/2 long-time tail is observed. For volume fractions from 0.45 to 0.48 the VAF becomes negative, before becoming positive and decaying with the positive 3/2 long-time tail. At the freezing volume fraction (0.494) the Alder 3/2 tail is not observed. At higher volume fractions a negative tail with an exponent of $5 / 2$ emerges, which coincides with the longtime tail of a Lorentz gas.
\end{abstract}

DOI: 10.1103/PhysRevLett.96.087801

PACS numbers: 61.20.Lc, 61.20.Ja, 64.70.Pf

One of the simplest statistical measures of atomic motion is the mean-squared displacement (MSD), $\left\langle\Delta \mathbf{r}_{i}^{2}\right\rangle$, constructed from the displacements $\Delta \mathbf{r}_{i}^{2}$ in a time interval averaged over an ensemble of atoms. When the motion is ballistic the MSD grows quadratically with $\tau$, whereas for diffusive motion the MSD grows linearly with $\tau$. A related and widely used statistical measure is the velocity autocorrelation function (VAF), $Z(\tau)$, defined by Ref. [1], $Z(\tau) \equiv \frac{1}{3}\left\langle\mathbf{v}_{i}(\tau) \cdot \mathbf{v}_{i}(0)\right\rangle=\frac{1}{6}\left(d^{2} / d \tau^{2}\right)\left\langle\Delta \mathbf{r}_{i}^{2}\right\rangle$. The dependence of the VAF on delay time more readily exposes motion that is neither ballistic nor diffusive. In this way $Z(\tau)$ gives a direct measure of the memory, or delay, associated with the disturbance created in the surrounding atomic fluid by the motion of an atom.

On the basis of Boltzmann's idea of uncorrelated binary collisions between the atoms in a fluid, one expects the VAF to decay exponentially with $\tau$ [1]. However, this notion was challenged with the discovery, in some of the earliest computer simulations, that $Z(\tau)$ decays algebraically at long delay times [2]. The shortcoming of the kinetic theory, as pioneered by Boltzmann, is that it fails to account for sequences of correlated collisions [3]. The circularity of such sequences, manifested in the continuum by a double vortex, gives a delayed impetus to an atom's initial velocity. While they may be rare, these sequences do occur and, moreover, they are the mechanism by which the transverse momentum diffuses. It is now well established [3-5] that, when fully developed, the influence of viscous flow is manifested by an algebraic decay of the VAF from above in proportion to $\tau^{-3 / 2}$, i.e., $Z(\tau)=$ $A \tau^{-3 / 2}$ where $A$ is the positive amplitude. Elastic modes will also affect how the VAF decays; such modes encompass (i) short wavelength propagating modes which, given the speed of sound in dense fluids, make no significant contribution to the VAF over the delay times associated with the development of the vorticity; and (ii) damped, long wavelength modes which incur delayed reversals of the atoms velocities [5-8].
As to the existence and origin of the so-called $\tau^{-3 / 2}$ "hydrodynamic tail," experiment $[9,10]$, computer simulation $[2,5-7,11,12]$, and theory $[3,4]$ are in complete accord. It has also been observed in both computer simulation $[5,6]$ and experiment $[13,14]$ that at high density the long wavelength longitudinal modes cause the VAF to become negative at intermediate delay times. However, there is no statistically compelling computer evidence to establish whether the VAF decays to zero from above or below at larger values of $\tau$ under these conditions. A related question that is possibly more interesting and important is whether elastic modes and viscous flow change their respective contribution or their character as the fluid is compressed or cooled beyond its freezing point.

To address these questions we have performed molecular dynamics computer simulations on a system of hard spheres. The new physics exposed by these computations is achieved in three ways: first by using a larger number of atoms and running the calculations longer than in previous work; second, by averaging a large number of simulation runs with different starting configurations; and third by using a coarse quasilogarithmic time scale.

The hard-sphere system has proved to be a valuable reference for the liquid state, and its equilibrium properties are well-known. In particular, it has been observed, in computer simulation [15] and experiment [16], that the system of hard spheres has a first order fluid to crystal transition; the fluid and (fcc) crystal coexist with the nominal volume fractions $\phi_{f}=0.494$ and $\phi_{m}=0.545$, respectively. Of course once the volume fraction exceeds, $\phi_{f}$, i.e., once the hard-sphere fluid is under-cooled, it crystallizes $[16,17]$ and it does so with increasing speed as the degree of under-cooling increases [18]. For the undercooled fluids we used an equimolar binary mixture of equal mass with a size ratio of 0.905 , which has nominal freezing and melting volume fractions of $\phi_{f}=0.506$ and $\phi_{m}=$ 0.548 [18]. This serves to delay the onset of crystallization long enough to observe the metastable fluid's dynamical 
behavior. In essence, to a good approximation the phase behavior of the binary or polydisperse system can be mapped onto that of a single component system. In a sense, this binary hard-sphere fluid is analogous to a colloidal fluid of hard spheres with a polydispersity of 5\%. Experiments on colloidal systems have shown that a modest degree of polydispersity causes a significant delay in crystallization without having a significant effect on dynamical properties such as the time correlation functions [19].

The data presented here was computed from simulations consisting of $N=10976$ particles. To check for finite size effects we repeated the calculations of the VAF at the highest $\phi=0.58$ and lowest $\phi=0.15$ volume fractions with $N=5 \times 10^{5}$ particles. There was no discernible systematic difference in the results for the two system sizes. We also calculated the speed of sound from the equation of state [20], which showed that finite size effects should be most important for the lowest volume fraction studied. For the larger $N=5 \times 10^{5}$ system it was found that a low frequency disturbance could not cross the periodic cell length on the longest time scale in our VAF calculations, regardless of volume fraction. Thus there can be little doubt that the results presented here are accurately representative of what would be obtained in the thermodynamic limit.

The VAFs were computed from 50 independent simulations, with a standard error in the region of the long-time tails of $4 \times 10^{-5}$, except those at the volume fractions shown in Fig. 4, which were computed from ensembles of 500 independent simulations, with a standard error of $1.5 \times 10^{-5}$.

Figure 1 shows the VAF versus $\log \tau$ for the one component fluid at several volume fractions in thermodynamic equilibrium $\left(\phi<\phi_{f}\right)$, at the freezing volume fraction $\left(\phi=\phi_{f}\right)$ and at a volume fraction for a marginally undercooled fluid $\left(\phi>\phi_{f}\right)$. The units used are such that the mean-squared thermal velocity, $Z(0)=k_{B} T / m=1$ and the time unit is $\sigma \sqrt{m / k_{B} T}$ where $T$ is the absolute temperature, $\sigma$ is the atomic diameter, $m$ is the atomic mass, and $k_{B}$ is Boltzmann's constant. From this plot it can be seen that the VAF becomes negative for $\phi \geq 0.45$.

In order to examine the long-time behavior, the data is replotted in Fig. 2 as a double logarithmic plot of $|Z(\tau)|$ versus $\tau$. From this figure it is clear that $Z(\tau)$ decays from the mean-squared thermal velocity, a condition imposed by definition, to an algebraic form consistent with $\tau^{-3 / 2}$, for all volume fractions below the freezing point, $\phi=0.494$. Thus, time coarse graining exposes a continuous and smooth crossover from ballistic motion to fully developed, viscous flow. The dip that becomes apparent for $\phi>0.3$ manifests velocity reversal incurred by damped compression modes, i.e., a transient visco-elastic response [8]. This transient response increases in strength with volume fraction and, for $\phi=0.45$, it causes $Z(\tau)$ to become negative, i.e., the velocities become anticorrelated at intermediate times. But even then $Z(\tau)$ crosses the abscissa again and decays to zero from above in a manner consistent with the

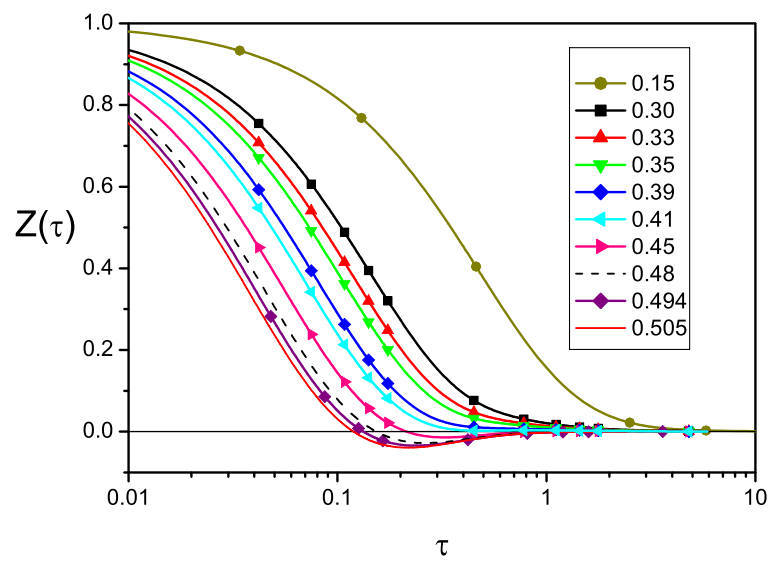

FIG. 1 (color online). A plot of the velocity autocorrelation function $Z(\tau)$ versus $\log \tau$ (symbols are defined in the legend), calculated from one component hard-sphere molecular dynamics simulations of fluids at various volume fractions $\phi=$ (volume of all the spheres divided by the total system volume). For $\phi \geq$ 0.45 the VAF becomes negative, so in order to expose the longtime behavior, double logarithmic plots of $|Z(\tau)|$ are needed (see Fig. 2).

power law, $\tau^{-3 / 2}$. Thus, for the fluid in thermodynamic equilibrium the data indicates that the fluid's delayed inelastic, viscous response to a thermally activated disturbance ultimately dominates, whatever the strength of its transient elastic response. This provides confirmation of what had previously only been conjectured $[8,21]$.

The data in Fig. 2 suggests that there is a qualitative change in behavior as the freezing point is approached, and

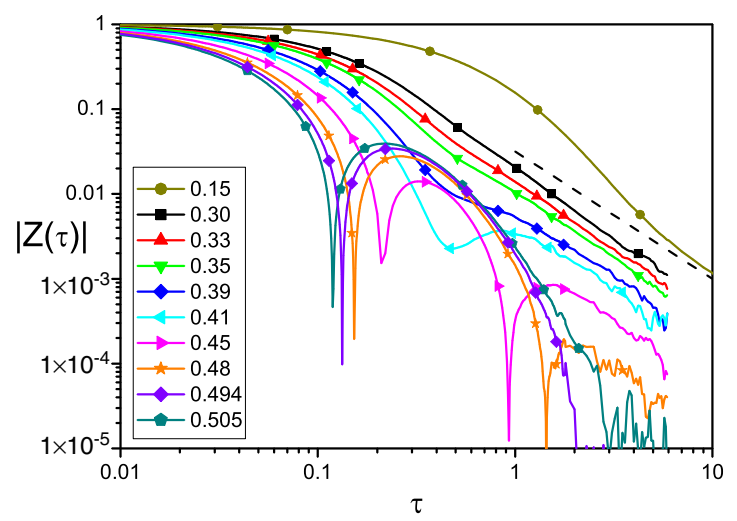

FIG. 2 (color online). A double logarithmic plot of $|Z(\tau)|$ for the data shown in Fig. 1. For all curves below $\phi_{f}=0.494$ a long-time $3 / 2$ power law (indicated by the dashed line) is clearly observed. As the volume fraction increases a nonmonotonic decay emerges as may be seen distinctly from the curve. We interpret this as indicative of the fluids strengthening viscoelastic behavior. For $\phi \geq 0.45$, the points where $Z(\tau)$ cross zero may be seen as a sharp minimum in this graph. For $\phi=$ 0.45 and $\phi=0.48$ a second sharp minimum is observed $[Z(\tau)$ has crossed zero again and now becomes positive], followed by a long-time $3 / 2$ tail. Upon increasing the volume fraction to $\phi=$ 0.494 and $\phi=0.505$ this reentrant positive behavior is no longer observed. 
this is also evident in the $\tau^{-3 / 2}$ tail. Although the time scale on which the $\tau^{-3 / 2}$ tail emerges does not change, the magnitude of the amplitude decreases dramatically, as shown in Fig. 3. Here we see that just below freezing the magnitude of the amplitude scales as $A \sim\left(\phi-\phi_{f}\right)^{1.5}$, which indicates that the amplitude goes to zero as the volume fraction approaches freezing.

In order to look more closely at the behavior near the freezing point, Fig. 4 shows the VAFs on a semilog scale for volume fractions just below freezing (0.480), at freezing (0.494), and just above freezing (0.505). At 0.48, the VAF recrosses the axis, as described above. However, at $\phi=0.494, Z(\tau)$ decays to zero from below by way of what appears to be an exponential function of delay time and a second crossing of the abscissa cannot be discerned from the noise. Moreover, at a volume fraction slightly above $\phi_{f}, \phi=0.505$, this decay appears slower than exponential, indicating the onset of a negative long-time tail.

The result for $\phi=0.505$ confirms that the second crossing point of the VAF disappears, as far as can be ascertained from our data, at $\phi_{f}$, and the VAF finally decays to zero from below for this volume fraction. In order to quantify this decay we need to undercool more deeply (i.e., go to higher volume fractions) which requires the use of the binary mixture [18].

In order to demonstrate the dynamical equivalence of the two systems, and highlight the fundamental change that our data shows upon traversal of the freezing transition, the VAFs for volume fractions from just below to just above this transition for the binary system are also shown in Fig. 4. As for the single component system, the volume fraction just below freezing (0.494) shows a second crossing point. However, there is no second crossing point observed for the freezing volume fraction $(0.506)$ or that just above freezing (0.513). This representation clearly

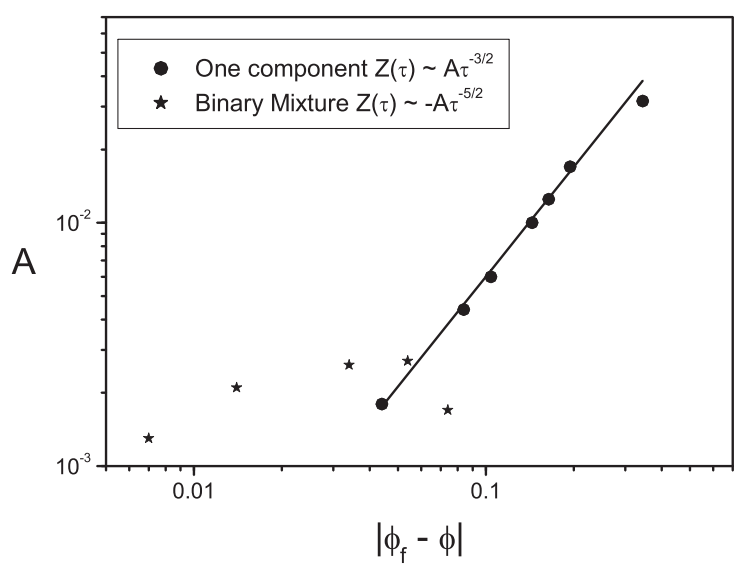

FIG. 3. Log-log plot of the amplitude $A$, as defined in the legend, as a function of $\left|\phi_{f}-\phi\right|$. The one component data is below freezing $\phi_{f}=0.494$ and the binary data is above freezing $\phi_{f}=0.506$. The solid line is a fit to the one component data $A=0.19\left(\phi_{f}-\phi\right)^{1.5}$, which goes to $A=0$ at freezing. demonstrates the fundamental nature of the change and shows that it applies for both the single component and the binary systems.

Having established that there is a fundamental change at or very close to the freezing volume fraction for both the single component and binary systems, we can now explore the under-cooled region more deeply. The VAFs for the under-cooled binary fluid as a function of volume fraction are shown in Fig. 5. By contrast to the equilibrium fluid, the VAFs for the under-cooled fluid all remain negative and decay to zero from below in a manner that can be described by the power law, $\tau^{-\mu}$, with $\mu=2.5$. The exponent has no significant volume fraction dependence while the amplitude of the power law has only a weak one. The most significant aspect of the data shown in Fig. 5 is that there is no evidence in the dynamical window of the reemergence of a positive algebraic decay, i.e., of fully developed vorticity. These results demonstrate a qualitative difference in dynamics between a thermodynamic equilibrium and metastable fluids.

A negative power-law decay has also been observed recently for an under-cooled colloidal fluid of hard spheres suspended in a liquid [13,14]. The difference between those results and the results presented here for the fluid of ballistic hard spheres is that the power-law index was found to be about $\mu=1.5$, and was weakly volume fraction dependent. Negative long-time tails for the VAF have also been found in the studies of fluids in confined geometries [22], where the development of vorticity is impaired, and longitudinal modes beyond a certain wavelength are over-damped. Moreover, as shown by computer studies [23], the presence of extremely long confining channels with nonslip walls results in over-damped longitudinal modes dominating the VAF at long times, causing it to decay algebraically from below. The index of the power laws for the colloidal hard spheres $[13,14]$ is consistent

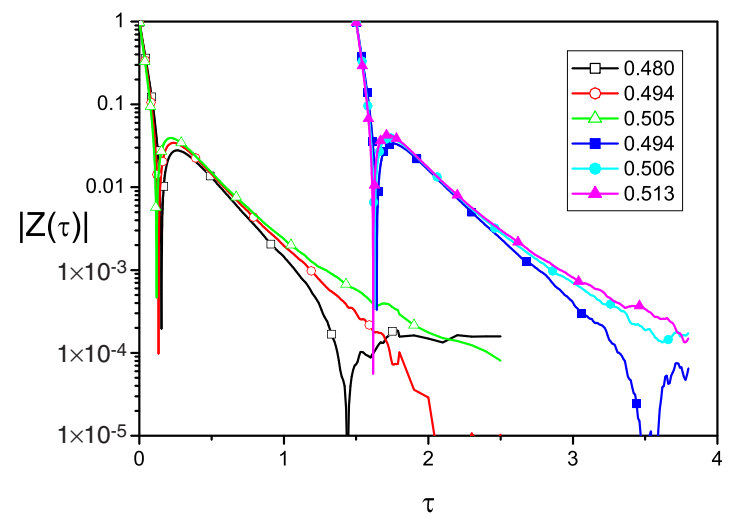

FIG. 4 (color online). A semilog plot of the absolute value of the VAF near the freezing volume fraction (symbols are defined in the legend) for both single component (open symbols) and binary systems (filled symbols). The squares are below $\phi_{f}$, the circles are nominally at $\phi_{f}$, and the triangles are just above $\phi_{f}$. The binary data is shifted to the right by 1.5 on the time scale for clarity. 


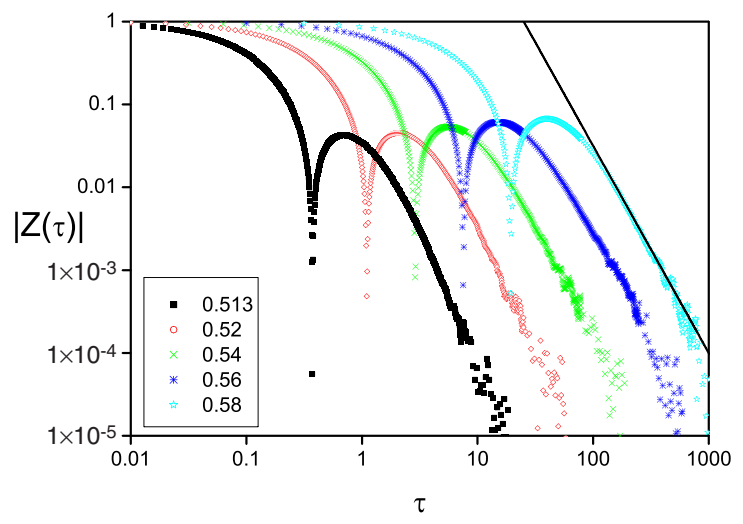

FIG. 5 (color online). A double logarithmic plot of the absolute value of the velocity autocorrelation function for a range of hard-sphere binary mixtures. For clarity, successive volume fractions have been shifted along the time axis by half a decade. A power law with exponent $5 / 2$ is also shown for the highest volume fraction.

with these studies. However, the index of the power law observed in the present work, $\mu=2.5$, coincides with the index for the negative tail of a three dimensional Lorentz gas [24].

Dynamical heterogeneities (which are both spatial and temporal in character) in under-cooled liquids have been observed in both simulations [25] and experiments [26] (for a review see Richert [27]). The spatial character of the heterogeneities manifests itself in multiple regions, which occupy a portion of the system's volume. On an appropriate time scale the particles inside these regions are immobile while the particles outside them are mobile. It is an interesting question as to whether these immobile regions play a similar role to the fixed scatterers of a Lorentz gas resulting in the $\mu=2.5$ power law observed here. The classical $\tau^{-3 / 2}$ tail requires local collective, linear momentum conservation combined with purely transverse momentum decay [18]. The dynamical heterogeneities are present over a longer time scale than the VAF can be discerned from the noise. Thus it is likely that the exchange of momentum between the mobile particles and the immobile regions is responsible for the breakdown of the classical $\tau^{-3 / 2}$ tail.

We conclude that for the hard-sphere fluid in thermodynamic equilibrium the long-time decay of the VAF can be described in terms of incompressible viscous flow leading to the emergence of the positive $\tau^{-3 / 2}$ tail. Our equilibrium simulations confirm this to be the case despite the fluid's elasticity. However, interestingly at the freezing point and in the under-cooled fluid the elasticity becomes so strong that the long-time tail associated with viscous flow can no longer be observed. This is probably due to the emergence of long-lived structural heterogeneities, which decay on a much slower time scale than the VAF. It may be that the slower decaying $\tau^{-3 / 2}$ tail reemerges at amplitudes too small for us to discern from the statistical noise. However, the dramatic decay in the amplitude of the $\tau^{-3 / 2}$ tail, upon approaching freezing as shown in Fig. 3, demonstrates that it is, at most, exceedingly small, and leaves open the possibility that it may indeed vanish at freezing.

This work was supported by the Victorian Partnership for Advanced Computing (VPAC).

[1] J. P. Hansen and I. R. McDonald, Theory of Simple Liquids (Academic Press, New York, 1991).

[2] B. J. Alder and T.E. Wainwright, Phys. Rev. A 1, 18 (1970).

[3] J. R. Dorfman and E. G. D. Cohen, Phys. Rev. A 6, 776 (1972).

[4] Y. Pomeau and P. Resibois, Phys. Rep. 19, 63 (1975).

[5] B. J. Alder, D. M. Gass, and T.E. Wainwright, J. Chem. Phys. 53, 3813 (1970).

[6] A. Rahman, Phys. Rev. 136, A405 (1964).

[7] D. Levesque and L. Verlet, Phys. Rev. A 2, 2514 (1970).

[8] R. Zwanzig and M. Bixon, Phys. Rev. A 2, 2005 (1970).

[9] J. P. Boon and A. Bouiller, Phys. Lett. 55A, 391 (1975).

[10] G. L. Paul and P. N. Pusey, J. Phys. A 14, 3301 (1981).

[11] D. Levesque and W. T. Ashurst, Phys. Rev. Lett. 33, 277 (1974).

[12] A. McDonough, S. P. Russo, and I. K. Snook, Phys. Rev. E 63, 026109 (2001).

[13] W. van Megen, J. Phys. Condens. Matter 14, 7699 (2002).

[14] W. van Megen Phys. Rev. E (to be published).

[15] W. G. Hoover and F. H. Ree, J. Chem. Phys. 47, 4873 (1967).

[16] P. N. Pusey and W. van Megen, Nature (London) 320, 340 (1986).

[17] B. O’Malley and I. Snook, Phys. Rev. Lett. 90, 085702 (2003).

[18] S. R. Williams, I. K. Snook, and W. van Megen, Phys. Rev. E 64, 021506 (2001).

[19] S. I. Henderson and W. van Megen, Phys. Rev. Lett. 80, 877 (1998).

[20] J. O. Hirschfelder, C. F. Curtiss, and R. B. Bird, Molecular Theory of Gases and Liquids (Wiley, New York, 1954), Eq. (3.B-7), p. 232.

[21] B.J. Alder, Stochastic Processes in Nonequilibrium Systems, Lecture Notes in Physics Vol. 84, edited by L. Garrido, P. Seglar, and P. J. Shepherd (SpringerVerlag, New York, 1978).

[22] M.H. J. Hagen, I. Pagonabarraga, C.P. Lowe, and D. Frenkel, Phys. Rev. Lett. 78, 3785 (1997).

[23] M. A. van der Hoef, D. Frenkel, and A. J. C. Ladd, Phys. Rev. Lett. 67, 3459 (1991).

[24] M. H. Ernst and A. Weyland, Phys. Lett. A 34, 39 (1971).

[25] M. M. Hurley and P. Harrowell, Phys. Rev. E 52, 1694 (1995).

[26] E. R. Weeks, J. C. Crocker, A. C. Levitt, A. Schofield, and D. A. Weitz, Science 287, 627 (2000).

[27] R. Richert, J. Phys. Condens. Matter 14, R703 (2002). 